Vipin Nair*, Rahul Thekkedath and Paduthol Godan Sankaran

\title{
The delayed effect of temperature on the risk of hospitalization due to COVID-19: evidence from Mumbai, India
}

https://doi.org/10.1515/em-2020-0039

Received September 12, 2020; accepted December 15, 2020; published online January 2, 2021

\section{Abstract}

Objectives: Meteorological factors and climatic variability have an immense influence on the transmission of infectious diseases and significantly impact human health. Present study quantifies the delayed effect of atmospheric temperature on the risk of hospitalization due to the Coronavirus disease 2019 (COVID-19) with adjusting the effects of other environmental factors in Mumbai, India.

Methods: The daily reported data of the number of hospitalized COVID-19 positive cases and the environmental factors at Mumbai, Maharashtra, India were collected and analyzed to quantify the main and the delayed effects. Exploratory data analysis and Distributed Linear and Non-linear lag Model (DLNM) with Generalized Additive Model (GAM) specification have applied to analyze the data.

Results: The study identified the Diurnal Temperature Range (DTR) delayed effect on the risk of hospitalization changed over the lag period of 0-14 days with increasing Relative Risk (RR) at the low DTR and decreasing RR at the higher DTR values. The extreme DTR suggests a high risk of hospitalization at earlier lags (i.e., 0-5 days). DTR's cumulative effect was significant at higher 0-10 lag days (p-value <0.05). Exposure to the low and moderate DTR suggests a high risk of hospitalization with more than six days of lag. The RR for daily average humidity with 95\% C.I was $0.996(0.967,1.027)$. The risk of hospitalization due to COVID-19 showed an increasing nature ( $p$-value $<0.05)$ with the increase in air pollution and average wind speed (WSAvg) at lag 0. Also, the risk of hospitalization changed through different lag periods of DTR. The analysis confirms the higher amount of delayed effect due to low DTR compared with moderate and high DTR.

Conclusions: The study suggests that both the climatic variations and air quality have significant impact on the transmission of the global pandemic COVID-19.

Keywords: air quality; COVID-19; diurnal temperature range; generalized additive model; Mumbai, India; wind speed.

\section{Introduction}

The global pandemic of Coronavirus disease (COVID-19) commenced from December 2019 in Wuhan, China, and has caused extreme havoc in the whole world. 2019-nCoV, commonly known as Coronavirus, is a novel highly contagious virus belonging to the Coronaviridae family that has been suspected to be transmitted to humans from animals and which had not previously been identified in humans. This virus causes mild to moderate respiratory illness and can lead to death in more severe cases. More than 215 countries and territories have been affected by COVID-19. COVID-19 had a dramatic impact on health care services and economies of affected countries.

\footnotetext{
*Corresponding author: Vipin Nair, Department of Data Science, Prasanna School of Public Health, Manipal Academy of Higher Education, Manipal, Karnataka, 576 104, India, E-mail: vipinn.stats@gmail.com. https://orcid.org/0000-0002-1449-7850

Rahul Thekkedath, Internal Debt Management Department, Reserve Bank of India, Mumbai, Maharashtra, India, E-mail: rahultstat@gmail.com Paduthol Godan Sankaran, Department of Statistics, Cochin University of Science and Technology, Kochi, Kerala, India, E-mail: sankaran.p.g@gmail.com
} 
All the nations across the globe are fighting against COVID-19 by slowing down the transmission of the virus since no proper medication is available at this time. The governments have been fighting against COVID-19 with their dual responsibility of public health and economic response to save lives and safeguard livelihoods. The authorities must balance public health interventions to control COVID-19 while seeking to minimize their social and economic impact. Governments have already implemented measures such as lockdowns and social distancing, with some positive results starting to emerge. Public health and social measures contribute to stopping individual chains of transmission and preventing outbreaks and are therefore critical in limiting further spread of COVID-19. The primary public health mandate to COVID-19 is expanding the health system capacity to save human life and developing vaccines as a clear exit strategy. At the same time, accurate research inputs backed by data and statistics are critical to the population, to quantify the infection fatality rate, and to identify factors affecting the transmission of the virus. Preparation and controlling the outbreak of COVID-19 diseases requires thorough planning and policies. Furthermore, during a pandemic, getting timely and accurate research insights is essential for taking effective countermeasures and reducing economic losses.

The modeling, estimation, and prediction of the prevalence of viruses and the epidemiological characteristics are important issues in providing the equipment needed to cope with their consequences and cutting the transmission chain of diseases (see, Funk et al. 2018; Jia et al. 2020; Woolhouse 2011 and the references cited therein). Some researchers have used statistical and mathematical modeling to forecast the confirmed cases and transmission risk of COVID-19 (see, Benvenuto et al. 2020; Maleki et al. 2020; Tang et al. 2020; Wu, Leung, and Leung 2020). Several studies have investigated the transmission dynamics of the COVID-19 pandemic (see, Li et al. 2020 and references cited therein). Huang et al. (2020) reported the epidemiological, clinical, laboratory, and radiological characteristics and treatment and clinical outcomes of the COVID-19 patients. Linton et al. (2020) investigated the incubation period and other time intervals that govern the epidemiological dynamics of COVID-19 infections.

The pandemic is reported to have spread through respiratory droplets and person-to-person contact (Li et al. 2020; Vellingiri et al. 2020). However, it might be possible that other unobserved environmental exposures may have facilitated the rate of the disease spreads through human-to-human transmission. Environmental factors like temperature and relative humidity may influence the transmissions of coronavirus (Casanova et al. 2010; Harmooshi, Shirbandi, and Rahim 2020) by affecting the survival of the virus in its transmission routes. There is evidence for the influence of environmental factors in the case of severe acute respiratory coronavirus (SARS-CoV) (Cai et al. 2007; Tan et al. 2005) and Middle East respiratory syndrome coronavirus (MERS-CoV) (Altamimi and Ahmed 2019; Gardner et al. 2019). The studies by Oliveiros et al. (2020), Wang et al. (2020a, 2020b), and Qi et al. (2020) reported an association of meteorological factors with COVID-19. Ma et al. (2020) examined the association between COVID-19 deaths and weather parameters in Wuhan, China. A spatiotemporal analysis by Briz-Redón and Serrano-Aroca (2020) for exploring the effect of temperature on COVID-19 early evolution found no evidence of a relationship between COVID-19 cases and temperature in Spain.

There exist high ambiguity about the role of climatic factors on the incidence of COVID-19 and little research in this direction. Modeling the association of the risk of hospitalization due to COVID-19 on a particular day with levels of environmental factors on the same day may not reveal the real association between exposure and outcome. The level of environmental factors reported in the past days may be more significant predictors for the risk of hospitalization at the present day than the current values of the covariates. In an environmental epidemiological study that models the association between the time-dependent explanatory variables and risk of hospitalization, it is vital to consider the delayed (lagged) associations between exposure and outcome Bhaskaran et al. (2013). The association between environmental temperature and COVID-19 cases from a global point of view without considering the delayed effect were investigated by Mandal and Panwar (2020). According to the United Nations, Mumbai, the capital city of the Indian state Maharashtra, is the second-most populous city in India after Delhi and the seventh most populous city in the world with a population more than 20 million. With over one-fifth of India's COVID-19 cases and a substantial increase in day-by-day, Mumbai is undoubtedly the country's biggest hotspot. Mumbai is explicitly having a tropical wet and dry climate. Using data of confirmed COVID-19 cases from the Mumbai district of Maharashtra, 
India, the present work mainly aims to identify the role of temperature on the risk of hospitalization due to COVID-19 after controlling the effects of factors such as humidity, time trends, air quality, and wind speed.

The contents of the present study are organized as follows. In Section 2, we describe the data and the methodology used for this study. The results of the statistical analysis are reported in Section 3. Finally, the concluding remarks for the present study with our significant findings are presented in Section 4.

\section{Methods}

\section{Data}

We consider the daily data of the number of hospitalized COVID-19 positive cases at Mumbai, Maharashtra, India, the most important affect place in India from the website of the Ministry of Health and Family Welfare, Government of India (www.mohfw. gov.in) and COVID-19 India (www.covid19india.org). We collected the data for the period March 11, 2020-May 07, 2020 (49 days). The information on metrological parameters for the study period obtained from the websites of the Central Pollution Control Board (CPCB), India, and the website of the weather underground (www.wunderground.com).

The main variables included in the study are the daily hospitalized counts denoted by $Y_{t}$ at day $t$, the Diurnal Temperature Range (DTR) measured in ${ }^{\circ}$ Fahrenheit, which is the difference between the daily maximum and minimum temperature, the daily average humidity (Havg) measured in \%, the time trends recorded in days, the daily Air Quality Index (AQI) and the daily average Wind Speed (WSAvg) measured in meter per hour.

\section{Statistical analysis}

The following statistical tools were mainly used for pursuing this study: i) exploratory data analysis including summary statistics and correlation analysis and 2) penalized Generalized Additive Models (GAM) with Distributed Linear and Non-linear lag Models (DLNM) introduced by Gasparrini, Armstrong, and Kenward (2010) for capturing the relationship of exposure and outcome and delayed effect (lag effect) of predictor variables on the outcome. Assuming the outcome $Y_{t} \sim \operatorname{negative} \operatorname{binomial}\left(\mu_{t}\right)$, with $\mu_{t} \equiv E\left(Y_{t}\right)$, the penalized GAM-DLNM model is defined by,

$$
\log \left(\mu_{t}\right)=\alpha+\boldsymbol{\beta} \operatorname{DTR}_{(t, l)}+\gamma \operatorname{Havg}_{t}+\mathrm{s}(\mathrm{AQI})+\mathrm{s}(\text { WSAvg })+\mathrm{s}(\text { day }),
$$

where, $t$ is the day of the observation; $\mu_{t}$ is the expected number of daily hospitalization for COVID-19 on day $t$; $\alpha$ is the intercept; $\operatorname{DTR}_{(t, l)}$ is a matrix obtained by applying the 'cross-basis' DLNM functions to DTR, $\boldsymbol{\beta}$ is the vector of coefficients for DTR $(t, l)$; and $l$ is the lag days. The natural cubic spline with default degrees of freedom was used to specify the exposure-outcome and lag-outcome relationships. Havg $_{t}$ is the reported value average humidity on day $t$ and $y$ is the corresponding regression coefficient. $\mathrm{s}($.$) denotes$ the penalized smoothing spline function. We used cubic regression spline with default degrees of freedom to control the effects of confounding factors, time trends of the day, air quality and daily WSAvg. All the statistical analyses were performed two-sided at a 5\% level of significance and conducted using R software (version 3.5.3) with the "mgcv" (version 1.8-317) and "dlnm" (version 2.4.1) packages.

\section{Results and discussion}

\section{Exploratory data analysis}

The summary statistics of the variables under consideration are reported in Table 1. A total number of 11,272 hospitalized cases were recorded at Mumbai due to COVID-19 from March 11, 2020, to May 07, 2020. Figure 1 depicts the pattern of daily hospitalized counts due to COVID-19 in Mumbai, India during the study period. The curve clearly shows the rapid growth in the hospitalized counts throughout the period. The Box-Whisker's plots depicted in Figure 2 explain the variability of hospitalized counts at different days of the week. The figure indicates the high variability and skewness of the data and confirms the presence of outlier observations. The variability of hospitalized counts in all other days except Wednesday and Friday is a little bit high comparing with the other days. 
Table 1: Summary statistics of daily weather conditions and daily hospitalization counts in Mumbai, India from 11-03-2020 to 07-05-2020 (58 days).

\begin{tabular}{lrrrrrrrr}
\hline Variables & Mean & SD & Mode & Min & \multicolumn{3}{c}{ Percentile } & \multicolumn{2}{c}{ Max } \\
\cline { 6 - 8 } & & & & & & $P_{25}$ & $P_{50}$ & $P_{75}$ \\
\hline Count & 194.34 & 225.39 & 1.00 & 0 & 6 & 112 & 369 & 769 \\
DTR & 11.50 & 3.66 & 9.00 & 6 & 9.00 & 11.00 & 14.00 & 22 \\
Huavg & 64.76 & 11.39 & 68.70 & 32.80 & 59.10 & 69.20 & 72.10 & 77.40 \\
WSAvg & 6.80 & 0.87 & 7.50 & 5 & 6.20 & 6.70 & 8 & 8.80 \\
AQI & 75.33 & 29.30 & 81.00 & 37 & 57 & 70 & 82 & 197 \\
\hline
\end{tabular}

DTR, Diurnal Temperature Range; WSAvg, Average Wind Speed; AQI, Air Quality Index.

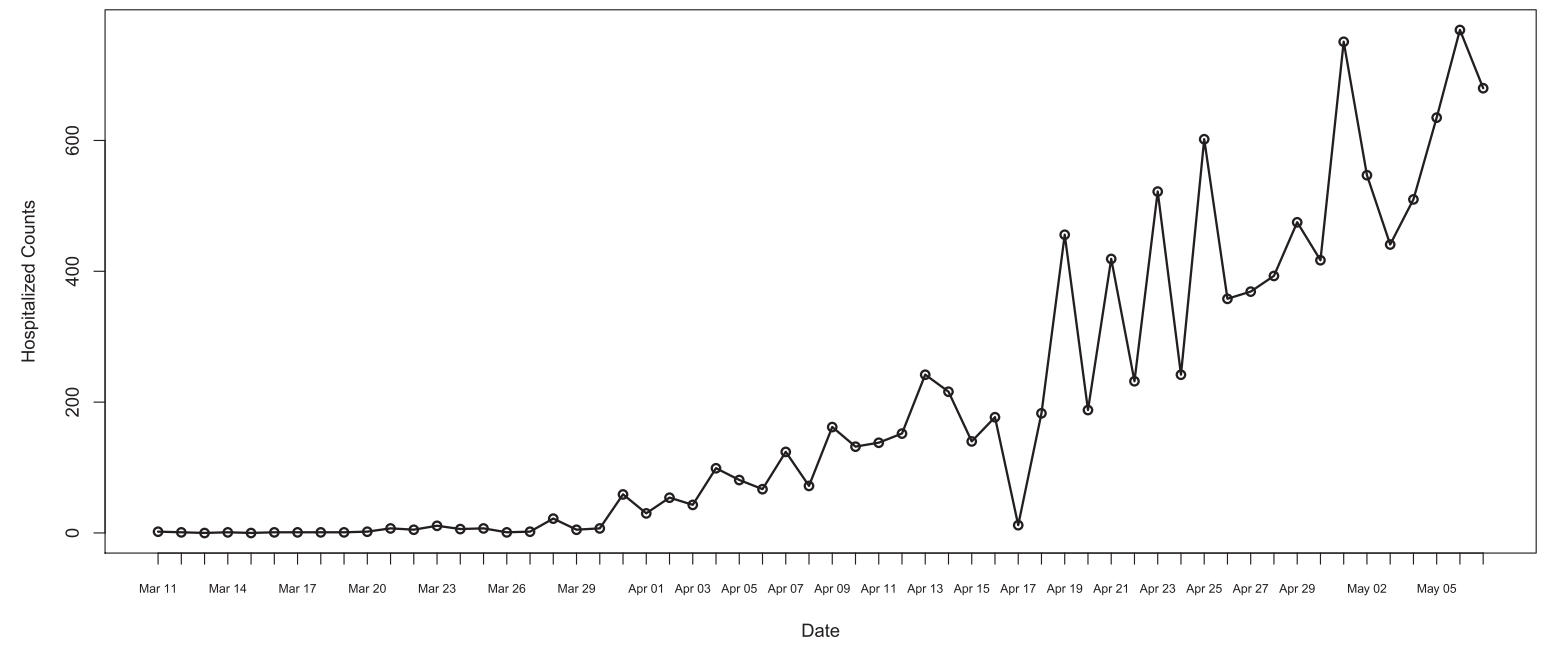

Figure 1: Time series plot of daily hospitalized counts due to COVID-19 in Mumbai, India from March 11, 2020 to May 07, 2020 (58 days).

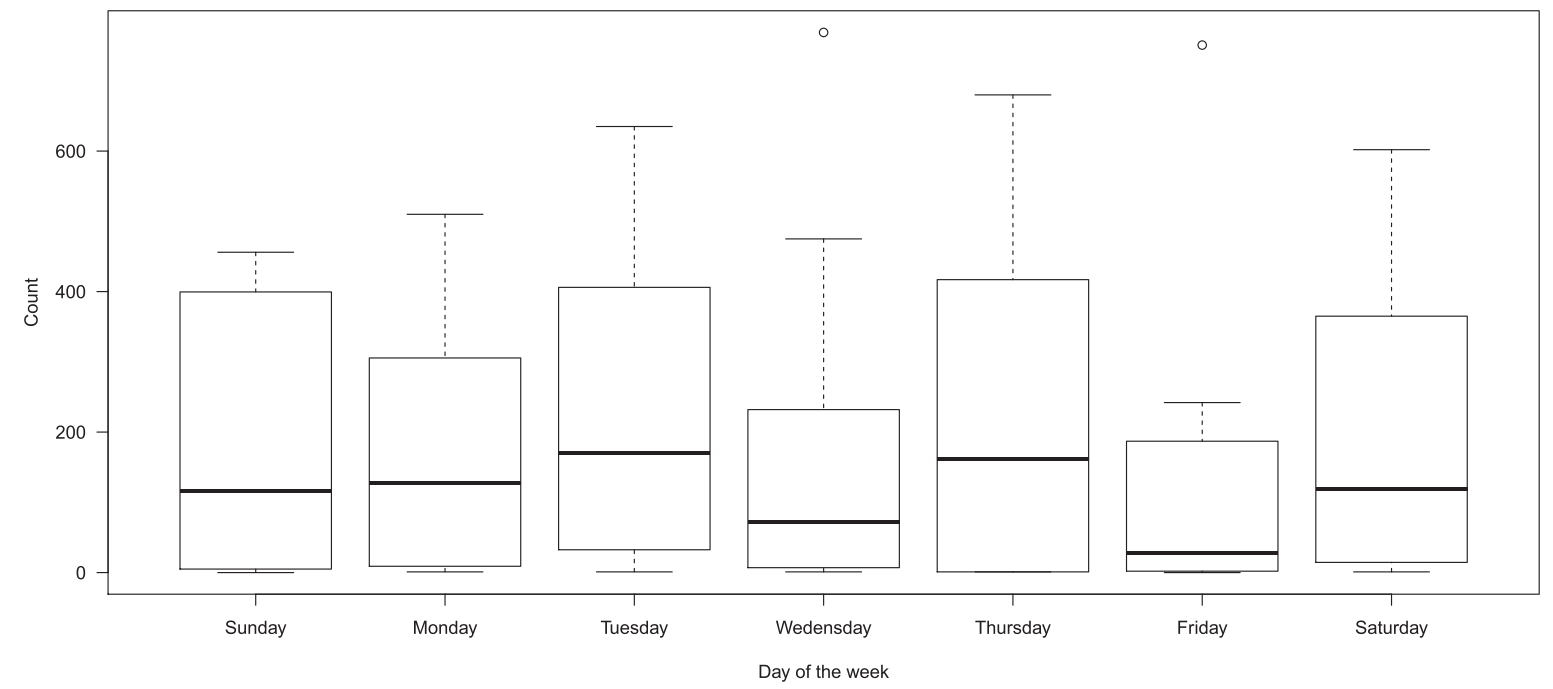

Figure 2: Box-Whiskers plots showing the variability of hospitalized count due to COVID-19 in Mumbai from March 11, 2020 to May 07, 2020 with Whiskers representing $\left(Q_{1}-1.5 \mathrm{IQR}, Q_{3}+1.5 \mathrm{IQR}\right)$. 


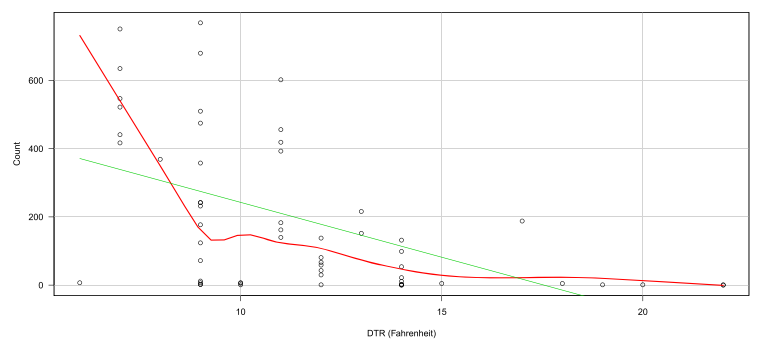

(a) Diurnal Temperature Range (DTR)

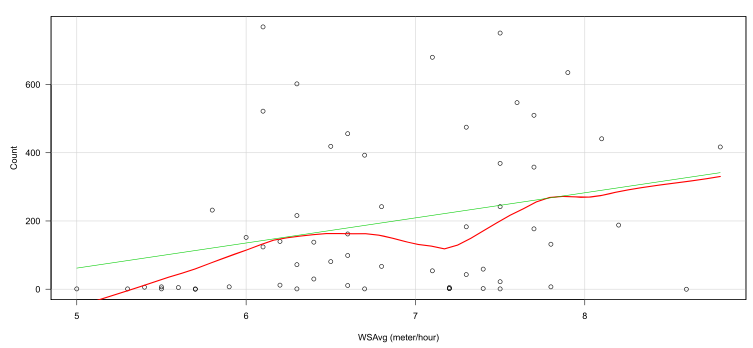

(c) Daily average wind speed

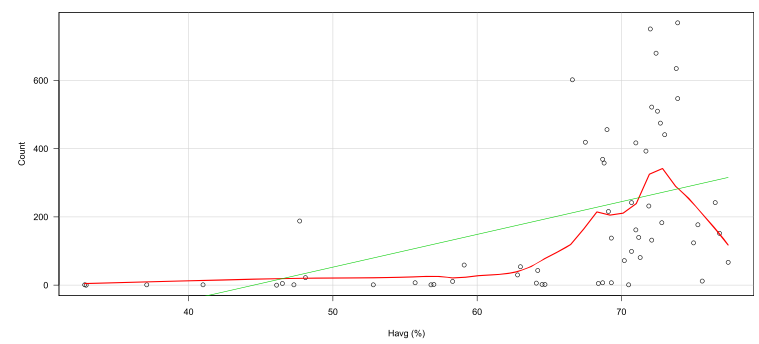

(b) Daily average humdity

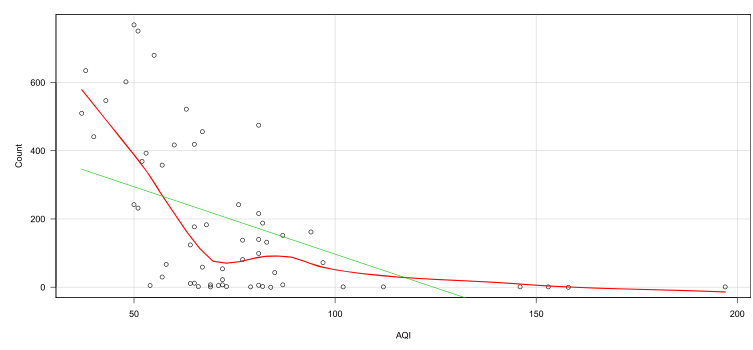

(d) Air Quality Index

Figure 3: Scatter plots of hospitalized counts and predictor variables. The green and red curves indicate the linear and non-linear relationships between the variables, respectively.

Spearman's non-parametric correlation between the counts and all other variables were estimated and tested at $5 \%$ of significance at Lag 0 (i.e., without considering the delayed effects). The results of the correlation analysis indicated that there exists a moderate significant association between DTR and daily reported hospitalized counts in the negative direction at Lag 0 ( $\hat{\rho}=-0.604$, $\mathrm{p}$-value $<0.001)$. Further, AQI is also showing a moderate significant negative relationship $(\hat{\rho}=-0.654$, p-value $<0.001)$. Both daily average humidity ( $\hat{\rho}=0.676$, $\mathrm{p}$-value $<0.001)$ and daily WSAvg $(\hat{\rho}=0.32$, $\mathrm{p}$-value $<0.001)$ show a moderately significant positive correlation with daily reported hospitalized counts. These relationships are graphically represented in Figure 3 using scatter plots. The plots confirm the presence of non-linear relationships between the outcome and the explanatory variables.

\section{Modeling the impact of diurnal temperature range}

Recently, Ma et al. (2020) identified among the Chinese population, the DTR has a positive association with COVID-19 spread. The summary statistics provided in Table 1 confirms the presence of overdispersion in the hospitalized count. We fitted a DLNM model with penalized GAM specification using negative binomial family and log link by Restricted Maximum Likelihood (REML) method of estimation. Eq. (2.1) specifies the general model. A maximum of 14 days of lag was considered to assess the relationship between DTR and hospitalized counts. The best model was identified according to the lowest of Akaike and Bayesian Information Criterion (AIC and BIC) associated with varying the degrees of freedom used to specify the spline functions for the additive components. The AIC and BIC of the fitted model were 514.14 and 534.62, respectively.

Figure 4 plots the relationship between the average humidity and other confounding variables with the risk of hospitalization. The daily average humidity shows a slight decreasing relationship (as depicted in Figure 4D) after adjusting for the effect of other variables. The adjusted relative risk (RR) of the daily average humidity is estimated at 95\% C.I was $0.996(0.967,1.027)$. The smooth additive term corresponds to s(day) was found to be statistically significant according to the chi-square test with effective degrees of freedom 2.628 


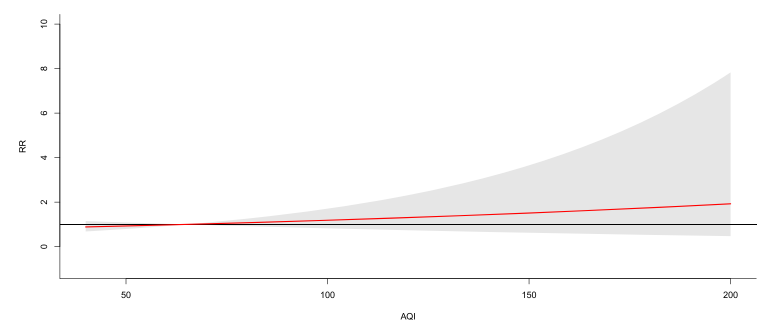

(a) Air quality

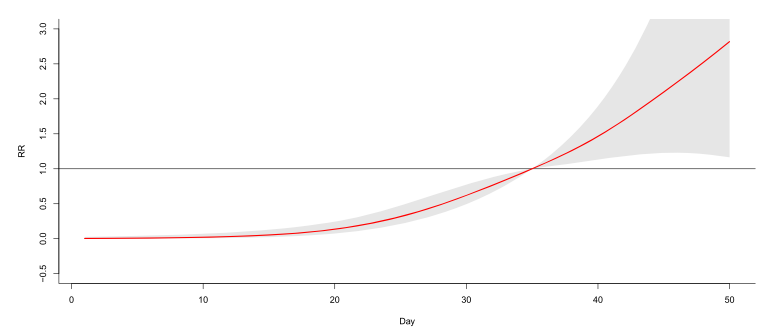

(c) Day

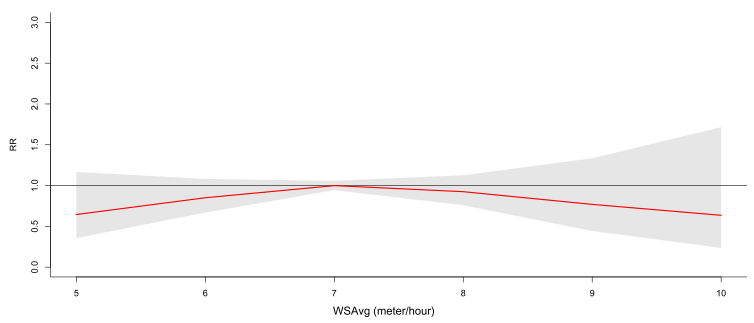

(b) Wind speed

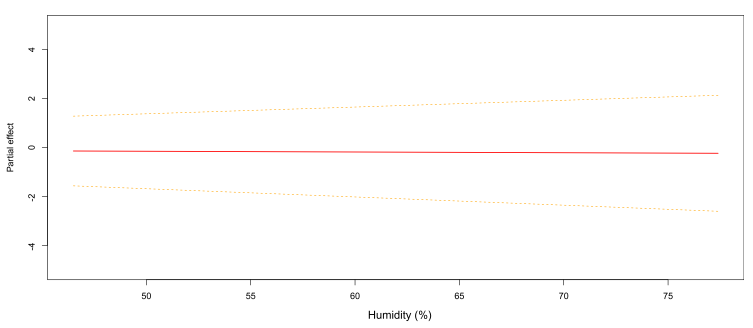

(d) Humidity

Figure 4: The estimated relationship between the predictor variables and the risk of hospitalization with $95 \% \mathrm{Cl}$. In (a) (b) and (c) the red lines are the relative risks, and grey regions are $95 \%$ confidence intervals. In (d) the red line is the estimated partial effect with $95 \%$ confidence interval.

(p-value $<0.001$ ). The risk of hospitalization due to COVID-19 shows a rapid increase until the end of the study period, as depicted in Figure 4C. Figure 4A indicates that the risk of hospitalization increases with increasing values of the AQI. The higher AQI indicates a higher amount of air pollution. Hence the figure confirms that there exists a high risk of COVID-19 spread through the contaminated air. Figure 4B indicates the chance of low risk of hospitalization concerning the daily WSAvg at Lag 0.

Figures 5 and 6 plots the effects for specific DTR values and delayed effects in terms of the lagged days. Figure 5 shows the RR by DTR at specific lags $(0,2,4,6,8,10,12$, and 14) and Figure 6 by lag at specific temperatures $\left(6,9,12,14,16,18,20\right.$, and $\left.22^{\circ} \mathrm{F}\right)$, corresponding to minimum to maximum of temperature distribution. The nature of the relationship between DTR and risk of hospitalization changes along with different lags. Figures 5 and 6 depict that till Lag 6, the risk of hospitalization shows an increasing nature with increasing DTR. After that, until Lag 14, the nature of the relationship is in the opposite direction. This plot confirms the delayed effect of low DTR compared with moderate and high DTR. The extreme DTR suggests a high risk of hospitalization at earlier lags till lag six days. The cumulative effect of DTR, adding up the contributions up to the 14 days of lag considered in the analysis, is included in Figure 7. The cumulative overall DTR effect on hospitalization also shows an increasing nature. The figure also confirms that at the cumulative of Lag 0-10 days, the lower DTR has significant RR (p-value <0.05) and larger values of DTR cause a high risk of hospitalization at earlier days of exposure.

All nations worldwide are facing severe economic and public health crises due to the global pandemic of COVID-19. The global pandemic is a severe public health emergency issue that commenced in December 2019 in Wuhan, China, caused extreme havoc in the whole world. Thorough planning and decision making backed by research is required to address this public health emergency and assess the health, social, and economic impact of COVID-19. Due to the absence of proper medication and vaccination against the pandemic, the crucial necessity for implementing suitable preventive health care strategies to reduce the transmission of the disease is one of the leading public health concerns the world now faces. As a developing nation with a higher rate of transmission and mortality and a lack of proper resources, India is one of the worst-hit nations due to COVID-19. This work studies the role of climatic conditions on the spread of the COVID-19 pandemic in Mumbai, India. 


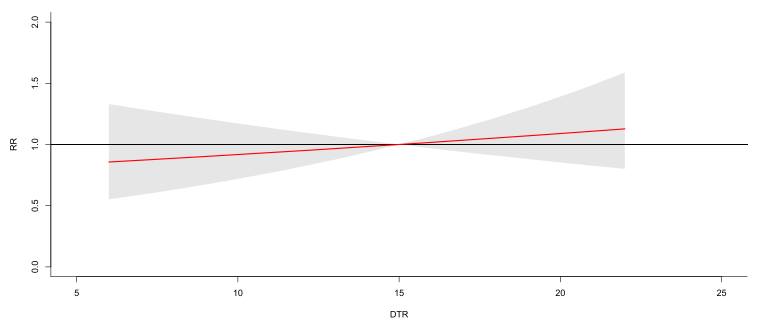

(a) $\operatorname{Lag}=0$

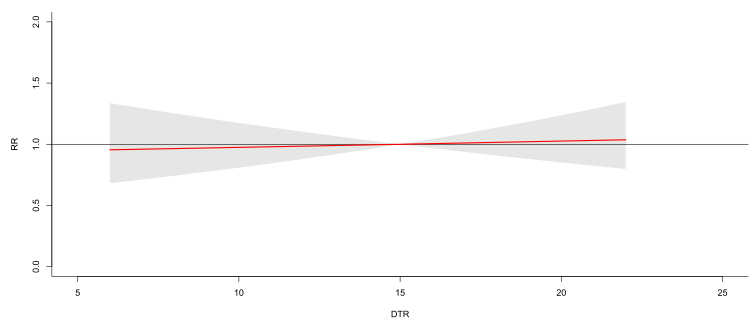

(c) $\operatorname{Lag}=4$

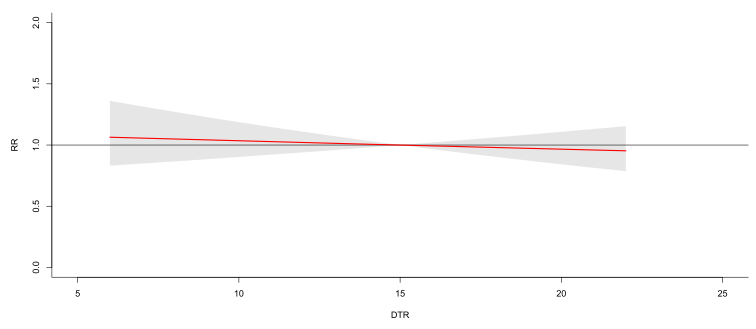

(e) $\operatorname{Lag}=8$

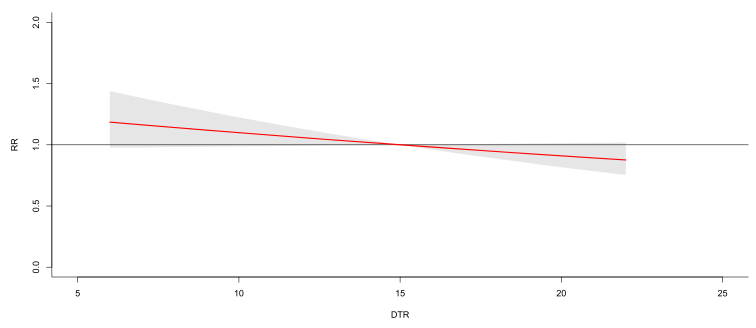

(g) $\operatorname{Lag}=12$

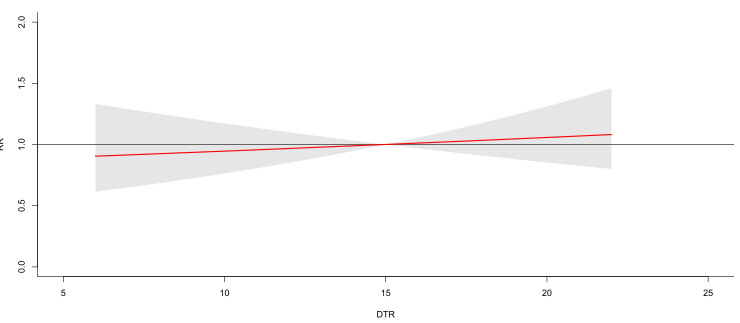

(b) $\operatorname{Lag}=2$

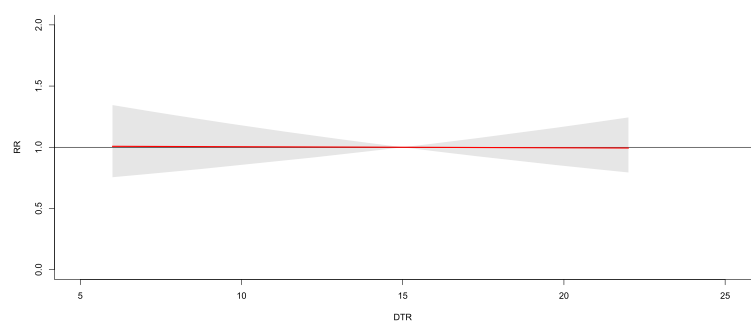

(d) $\operatorname{Lag}=6$

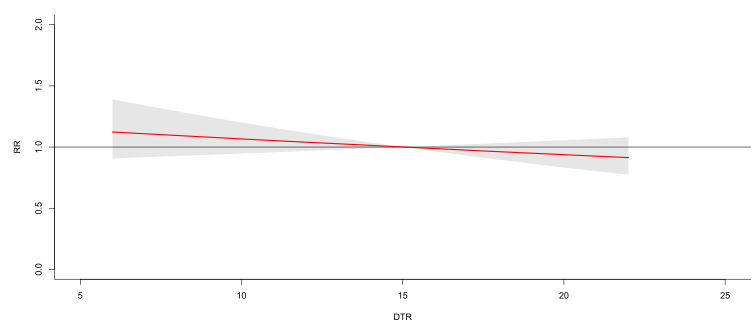

(f) $\operatorname{Lag}=10$

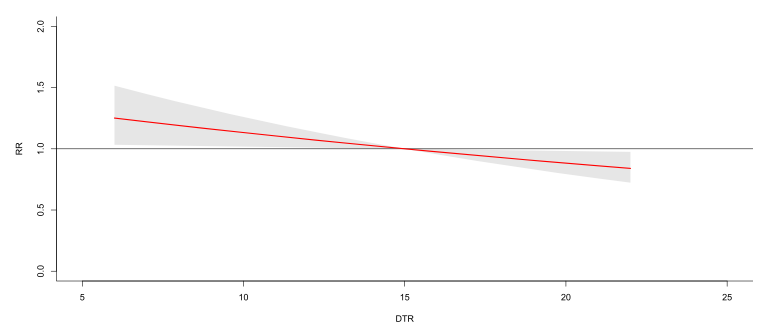

(h) $\operatorname{Lag}=14$

Figure 5: Plot of relative risks by DTR at specific lags. Red lines are the relative risks, and grey regions are $95 \%$ confidence intervals.

In order to plan and implement new policies and strategies against the global pandemic due to the infectious disease, it is vital to identify the influence of the exposures on the transmission of the disease. Apart from personal level health characteristics, environmental factors also play a crucial role in the spread of COVID-19. Climate change and variability in the levels of meteorological factors have a significant association with the transmission of infectious diseases in India Dhara, Schramm, and Luber (2013). One important criterion to consider while modeling this exposure-outcome relationship is the role of the significant delayed effect of time-varying predictors on the risk of hospitalization due to COVID-19. 


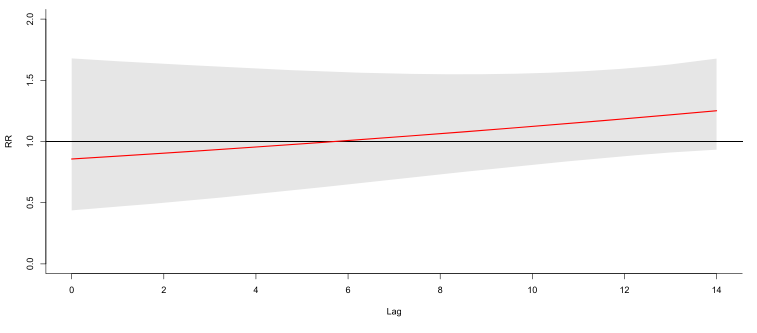

(a) $\mathrm{DTR}=6($ Minimum $)$

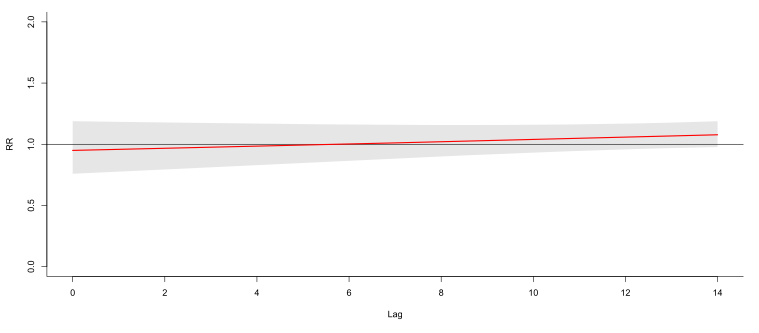

(c) $\mathrm{DTR}=12\left(P_{50}\right)$

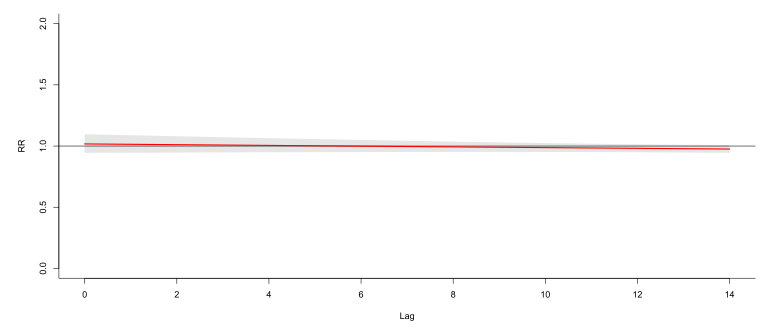

(e) DTR $=16$

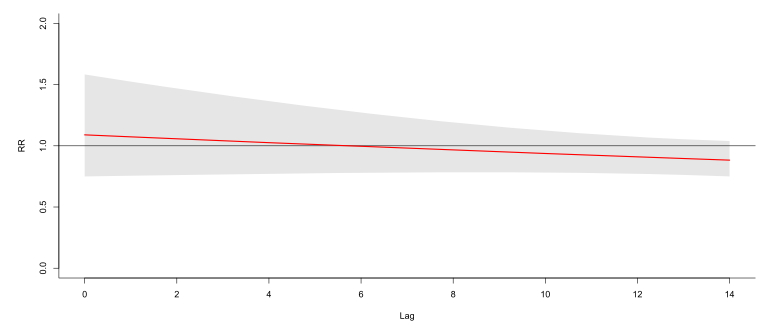

(g) DTR $=20$

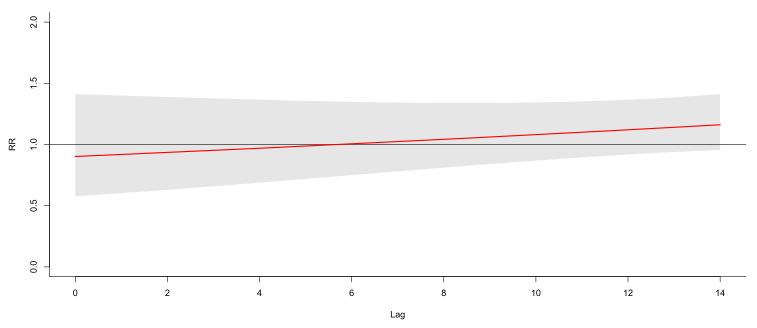

(b) $\mathrm{DTR}=9\left(P_{25}\right)$

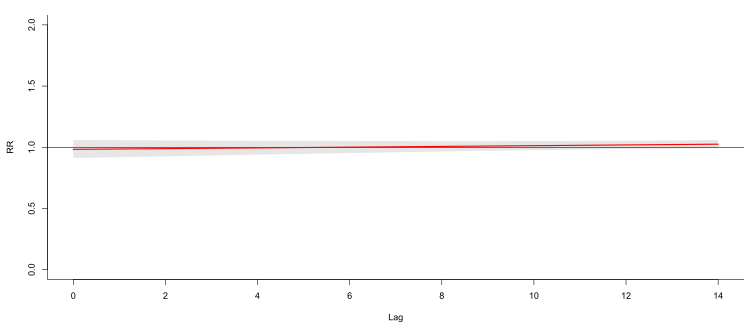

(d) DTR $=14\left(P_{75}\right)$

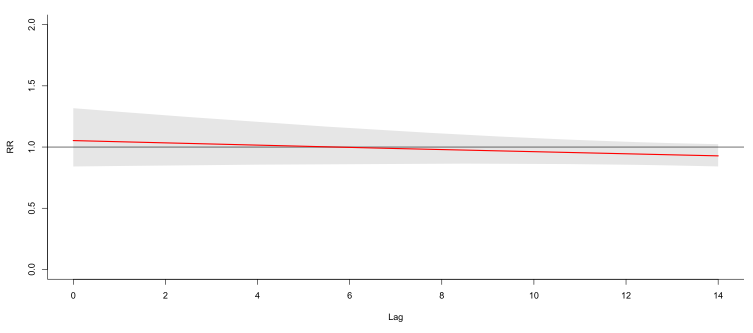

(f) $\mathrm{DTR}=18$

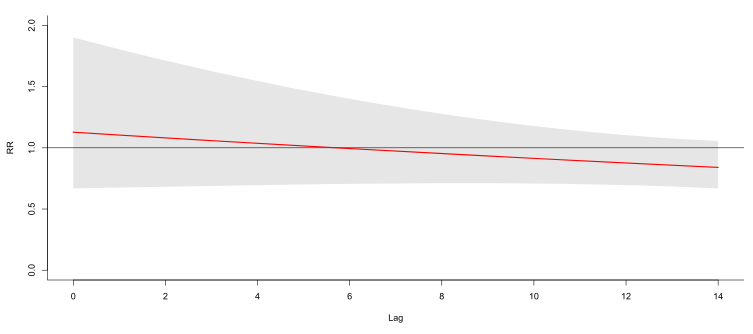

(h) DTR $=22($ Maximum $)$

Figure 6: Plot of relative risks by lags at specific DTR. Red lines are the relative risks, and grey regions are $95 \%$ confidence intervals.

In the present study, we explored and exploited the delayed effect of DTR on the risk of hospitalization due to COVID-19 after adjusting for other environmental factors. In the Indian population, this is the first study to investigate the delayed effect of atmospheric temperature on the spread of COVID-19. Our findings show high variability in daily reported COVID-19 positive cases in Mumbai, India's financial city. The correlation analysis results without considering the delayed effects identified the reported hospitalized COVID-19 cases with the daily average humidity and daily WSAvg had a significant moderate positive correlation. DTR and daily air 


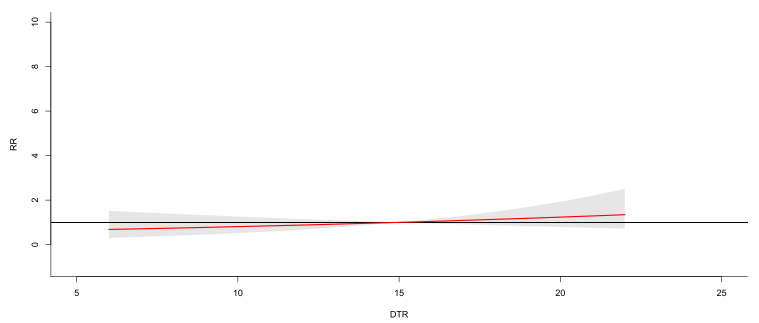

(a) Lag 0-2

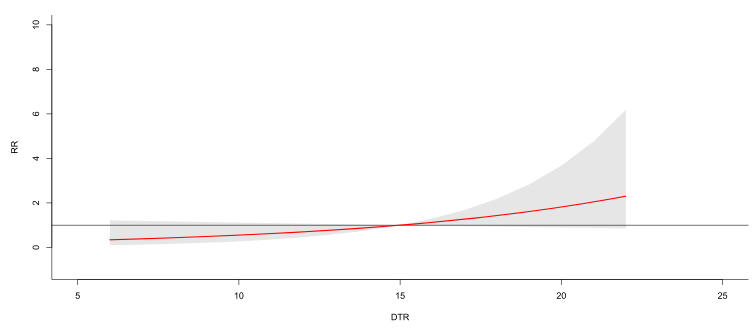

(c) Lag 0-7

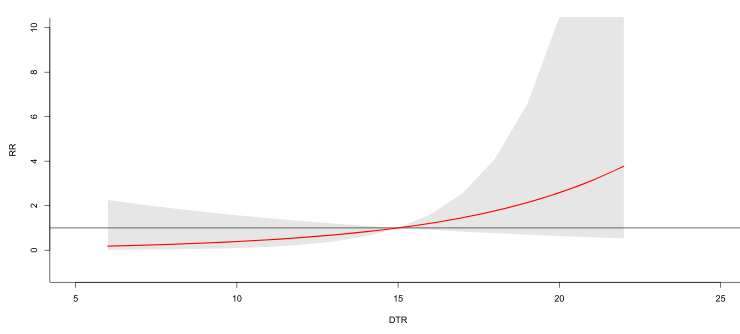

(e) Lag 0-12

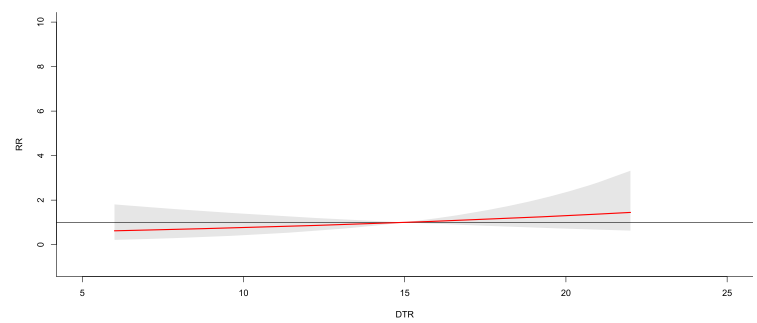

(b) Lag 0-5

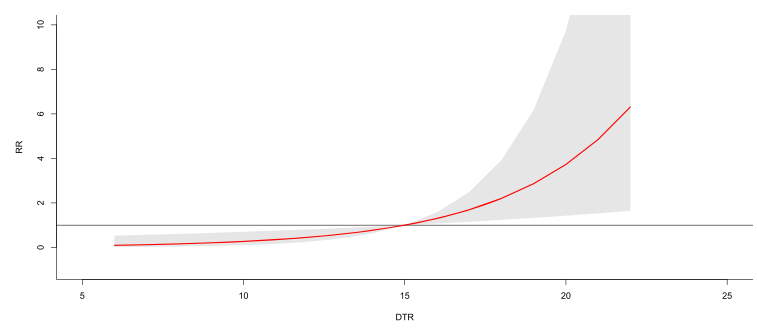

(d) Lag 0-10

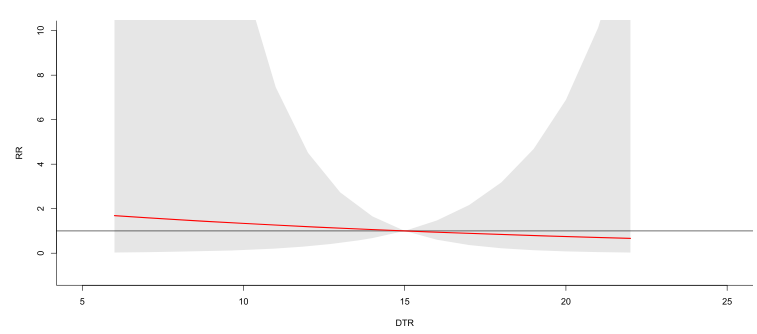

(f) Lag 0-14

Figure 7: Cumulative overall DTR effects on hospitalization at different lag periods. Red lines are the cumulative relative risks, and grey regions are $95 \%$ confidence intervals.

quality had a significant negative correlation. Further, the results of the DLNM analysis revealed that the delayed effect of the DTR on the risk of hospitalization changed over the lag period of 0-14 days.

\section{Conclusion}

The present study indicates a relatively high risk of hospitalization concerning highly polluted air. Further research is required to quantify air quality's delayed effect on the risk of hospitalization due to COVID-19. We estimated the RR to measure the overall cumulative effects associated with exposure to DTR's specific values at the earlier values of delayed effects at different lag days. The empirical evidence reveals that the overall increase in the hospitalizes counts after a constant exposure to specific DTR sustained throughout the earlier lag period 2 or 5 days (backward perspective) or as the sum of the contributions of exposure to a specific DTR in the next 2 or 5 days (forward perspective). The analysis confirms the higher amount of delayed effect due to low DTR compared with moderate and high DTR. The extreme DTR suggests a significantly high risk of hospitalization at earlier lags (i.e., $0-5$ days). In contrast, exposure to the low and moderate DTR suggests a high risk of hospitalization with more than six days of lag. The cumulative effect of DTR on the risk of hospitalization due to 
COVID-19 showed an increasing nature over the lag of 0-12 days. Moreover, this cumulative effect was statistically significant at a lag of 0-10 days.

An essential finding of this study is the significant association of DTR and the number of hospitalized COVID-19 cases in Mumbai, India. Our findings show that the RR of hospitalization changed through lag periods and concerning DTR. Exposure to high DTR causes early admission and low DTR may delay the risk of hospitalization due to COVID-19. This study suffers from the limitation of early case investigation. Within this limitation, the study suggests that the variation in daily temperature and change in levels of meteorological factors also have a significant role in the COVID-19 transmission in Mumbai, India.

Acknowledgments: The authors would like to express their gratitude to the Editor and the anonymous reviewers for their constructive comments, suggestions, and careful reading of the manuscript that improved the presentation and the contents.

Research funding: None declared.

Author contributions: VN analyzed and interpreted the data regarding the COVID-19 disease and was a major contributor in writing the manuscript. RT prepared the data for the analysis. PGS contributed in the interpretation of the analysis results. All authors read and approved the final manuscript.

Competing interests: The authors declare that they have no competing interests.

Informed consent: Not applicable.

Ethical approval: A secondary freely available data released by the Government of India was used for the analysis. The data set had no identifiable evidence on the survey participants, therefore ethical approval is not required for this work.

Availability of data and materials: The datasets used and/or analyzed during the current study are available from the corresponding author on reasonable request.

Disclaimer: The views expressed in the article are those of the authors and do not represent the views of the organization in which authors belong.

\section{References}

Altamimi, A., and A. E. Ahmed. 2019. “Climate Factors and Incidence of Middle East Respiratory Syndrome Coronavirus.” Journal of Infection and Public Health 13 (5): 704-8.

Benvenuto, D., M. Giovanetti, L. Vassallo, S. Angeletti, and M. Ciccozzi. 2020. “Application of the Arima Model on the Covid-2019 Epidemic Dataset." Data in Brief 29: 105340.

Bhaskaran, K., A. Gasparrini, S. Hajat, L. Smeeth, and B. Armstrong. 2013. "Time Series Regression Studies in Environmental Epidemiology.” International Journal of Epidemiology 42 (4): 1187-95.

Briz-Redón, Á., and Á. Serrano-Aroca. 2020. "A Spatio-Temporal Analysis for Exploring the Effect of Temperature on Covid-19 Early Evolution in spain." Science of The Total Environment 728: 138811.

Cai, Q.-C., J. Lu, Q.-F. Xu, Q. Guo, D.-Z. Xu, Q.-W. Sun, H. Yang, G.-M. Zhao, and Q.-W. Jiang. 2007. "Influence of Meteorological Factors and Air Pollution on the Outbreak of Severe Acute Respiratory Syndrome." Public Health 121 (4): 258-65.

Casanova, L. M., S. Jeon, W. A. Rutala, D. J. Weber, and M. D. Sobsey. 2010. "Effects of Air Temperature and Relative Humidity on Coronavirus Survival on Surfaces.” Applied and Environmental Microbiology 76 (9): 2712-7.

Dhara, V. R., P. J. Schramm, and G. Luber. 2013. “Climate Change \& Infectious Diseases in India: Implications for Health Care Providers." Indian Journal of Medical Research 138 (6): 847.

Funk, S., A. Camacho, A. J. Kucharski, R. M. Eggo, and W. J. Edmunds. 2018. "Real-time Forecasting of Infectious Disease Dynamics with a Stochastic Semi-Mechanistic Model." Epidemics 22: 56-61.

Gardner, E. G., D. Kelton, Z. Poljak, M. Van Kerkhove, S. von Dobschuetz, and A. L. Greer. 2019. "A Case-Crossover Analysis of the Impact of Weather on Primary Cases of Middle East Respiratory Syndrome." BMC Infectious Diseases 19 (1): 113.

Gasparrini, A., B. Armstrong, and M. G. Kenward. 2010. “Distributed Lag Non-linear Models.” Statistics in Medicine 29 (21): 2224-34.

Harmooshi, N. N., K. Shirbandi, and F. Rahim. 2020. "Environmental Concern Regarding the Effect of Humidity and Temperature on 2019-ncov Survival: Fact or Fiction.” Environmental Science and Pollution Research 27: 1-10. 
Huang, C., Y. Wang, X. Li, L. Ren, J. Zhao, Y. Hu, L. Zhang, G. Fan, J. Xu, X. Gu, Z. Cheng, T. Yu, J. Xia, Y. Wei, W. Wu, X. Xie, W. Yin, H. Li, M. Liu, Y. Xiao, H. Gao, L. Guo, J. Xie, G. Wang, R. Jiang, Z. Gao, Q. Jin, J. Wang, and B. Cao. 2020. "Clinical Features of Patients Infected with 2019 Novel Coronavirus in Wuhan, China." The Lancet 395 (10223): 497-506.

Jia, L., K. Li, Y. Jiang, X. Guo, and T. Zhao. 2020. "Prediction and Analysis of Coronavirus Disease 2019." arXiv preprint arXiv: 2003.05447.

Li, Q., X. Guan, P. Wu, X. Wang, L. Zhou, Y. Tong, R. Ren, K. S. Leung, E. H. Lau, J. Y. Wong, X. Xing, N. Xiang, Y. Wu, C. Li, Q. Chen, D. Li, T. Liu, J. Zhao, M. Liu, W. Tu, C. Chen, L. Jin, R. Yang, Q. Wang, S. Zhou, R. Wang, H. Liu, Y. Luo, Y. Liu, G. Shao, H. Li, Z. Tao, Y. Yang, Z. Deng, B. Liu, Z. Ma, Y. Zhang, G. Shi, T. T. Lam, J. T. Wu, G. F. Gao, B. J. Cowling, B. Yang, G. M. Leung, and Z. Feng. 2020. “Early Transmission Dynamics in Wuhan, China, of Novel Coronavirus-Infected Pneumonia." New England Journal of Medicine 382: 1199-207.

Linton, N. M., T. Kobayashi, Y. Yang, K. Hayashi, A. R. Akhmetzhanov, S.-M. Jung, B. Yuan, R. Kinoshita, and H. Nishiura. 2020. "Incubation Period and Other Epidemiological Characteristics of 2019 Novel Coronavirus Infections with Right Truncation: A Statistical Analysis of Publicly Available Case Data." Journal of Clinical Medicine 9 (2): 538.

Ma, Y., Y. Zhao, J. Liu, X. He, B. Wang, S. Fu, J. Yan, J. Niu, J. Zhou, and B. Luo. 2020. "Effects of Temperature Variation and Humidity on the Death of Covid-19 in Wuhan, China." The Science of the Total Environment 724: 138226.

Maleki, M., M. R. Mahmoudi, D. Wraith, and K.-H. Pho. 2020. "Time Series Modelling to Forecast the Confirmed and Recovered Cases of Covid-19." Travel Medicine and Infectious Disease 37: 101742.

Mandal, C. C., and M. Panwar. 2020. “Can the Summer Temperature Drop Covid-19 Cases?” Public Health 185: 72-9.

Oliveiros, B., L. Caramelo, N. C. Ferreira, and F. Caramelo. 2020. "Role of Temperature and Humidity in the Modulation of the Doubling Time of Covid-19 Cases.” medRxiv https://doi.org/10.1101/2020.03.05.20031872.

Qi, H., S. Xiao, R. Shi, M. P. Ward, Y. Chen, W. Tu, Q. Su, W. Wang, X. Wang, and Z. Zhang. 2020. "Covid-19 Transmission in Mainland China is Associated with Temperature and Humidity: A Time-Series Analysis." Science of the Total Environment 728: 138778.

Tan, J., L. Mu, J. Huang, S. Yu, B. Chen, and J. Yin. 2005. "An Initial Investigation of the Association between the Sars Outbreak and Weather: With the View of the Environmental Temperature and its Variation." Journal of Epidemiology \& Community Health 59 (3): 186-92.

Tang, B., X. Wang, Q. Li, N. L. Bragazzi, S. Tang, Y. Xiao, and J. Wu. 2020. "Estimation of the Transmission Risk of the 2019-ncov and its Implication for Public Health Interventions.” Journal of Clinical Medicine 9 (2): 462.

Vellingiri, B., K. Jayaramayya, M. Iyer, A. Narayanasamy, V. Govindasamy, B. Giridharan, S. Ganesan, A. Venugopal, D. Venkatesan, H. Ganesan, K. Rajagopalan, P. K. S. M Rahman, S Cho, N. S. Kumar, and M. D. Subramaniam. 2020. “Covid-19: A Promising Cure for the Global Panic." Science of the Total Environment 725: 138277.

Wang, C., P. W. Horby, F. G. Hayden, and G. F. Gao. 2020a. “A Novel Coronavirus Outbreak of Global Health Concern.” The Lancet 395 (10223): 470-3.

Wang, M., A. Jiang, L. Gong, L. Luo, W. Guo, C. Li, J. Zheng, C. Li, B. Yang, J. Zeng, Y. Chen. 2020b. “Temperature Significant Change Covid-19 Transmission in 429 Cities." MedRxiv.

Woolhouse, M. 2011. "How to Make Predictions about Future Infectious Disease Risks." Philosophical Transactions of the Royal Society B: Biological Sciences 366 (1573): 2045-54.

Wu, J. T., K. Leung, and G. M. Leung. 2020. "Nowcasting and Forecasting the Potential Domestic and International Spread of the 2019-ncov Outbreak Originating in Wuhan, China: A Modelling Study.” The Lancet 395 (10225): 689-97. 